\title{
Fast-Fourier-based error propagation for the gravimetric terrain correction
}

\author{
J. C. McCubbine ${ }^{1}$, W. E. Featherstone ${ }^{1}$, and J. F. Kirby ${ }^{1}$
}

\begin{abstract}
We have identified a gap in the literature on error propagation in the gravimetric terrain correction. Therefore, we have derived a mathematical framework to model the propagation of spatially correlated digital elevation model errors into gravimetric terrain corrections. As an example, we have determined how such an error model can be formulated for the planar terrain correction and then be evaluated efficiently using the 2D Fourier transform. We have computed 18.3 billion linear terrain corrections and corresponding error estimates for a 1 arc-second ( $\sim 30 \mathrm{~m})$ digital elevation model covering the whole of the Australian continent.
\end{abstract}

\section{INTRODUCTION}

The gravimetric terrain correction is added to the simple Bouguer gravity anomaly to produce the refined or complete Bouguer gravity anomaly. This correction accounts for the departures of the Earth's topography from a plate, shell, or cap model (e.g., Nowell, 1999). Most existing literature on the gravimetric terrain correction has focused on formulas for various models of the topography (e.g., mass-prism, mass-line, polyhedron, or smoother models [e.g., $\mathrm{Wu}$, 2016]) with differing levels of complexity. However, no universally accepted methodology exists to determine the errors propagated into calculated terrain corrections from digital elevation model (DEM) height errors.

Dransfield and Zeng (2009) investigate errors in terrain corrections as a result of DEM height errors for airborne gravity gradient measurements. However, only empirical tests and gross simplifications have been explored to obtain approximate error estimates for the gravimetric terrain corrections: Mikuška et al. (2006) show that long-wavelength biases in DEMs introduce substantial uncertainties at the continental scale; Steinhauser et al. (1990) demonstrate the effect of restricting the computational extent; Zahorek et al. (2010) empirically explore the effects of using different terrain-correction formulas, inaccuracies in the computation points, and the effect of using different DEMs, showing the accuracy of the latter to have the most significant effect.

Malgorzata and Krynski (2009) analytically investigate errors in terrain corrections introduced by mislocating the computation points and intrinsic inaccuracies in the DEM. They present a simplified linear formula based on the DEM height error and the Bouguer plate formula, which is sufficient in flat terrain. However, their formula neglects to incorporate the dependence of the error on the coarseness of the topography in rougher terrains, and this overlooks the law of error propagation in the terrain-correction integral formula.

We therefore derive error propagation formulas for planar, firstorder terrain corrections as being dependent on the coarseness of the local topography and spatially correlated errors in the DEM heights. We consider that the coordinates of the computation points are error free because they are grid-node regist"rations and not the gravity observation location (unlike Malgorzata and Krynski, 2009). The principles discussed here can be applied to other terrain-correction formulas, or to other convolution integrals. We fast Fourier transform the convolution integrals to the frequency domain for efficient evaluation. As a numerical example, we generate a $1^{\prime \prime} \times 1^{\prime \prime}(\sim 30 \mathrm{~m})$ grid of planar terrain corrections and the associated error estimates over the whole of the Australian continent.

\section{PLANAR TERRAIN CORRECTIONS OVER AUSTRALIA}

Previous grids of planar terrain corrections over the whole of Australia (Kirby and Featherstone, 1999, 2002) used the $9^{\prime \prime} \times 9^{\prime \prime}$ ( $\sim 250 \mathrm{~m}$ ) resolution GEODATA DEMs produced by Geoscience Australia. The release of $1^{\prime \prime} \times 1^{\prime \prime}$ resolution DEMs from the shuttle radar topography mission (SRTM) (Farr et al., 2007) warrants for the recomputation of terrain corrections at this higher spatial resolution. We only consider the planar terrain correction here because Kuhn et al. (2009) show that complete planar Bouguer anomalies (with the Bouguer slab given by $2 \pi G \rho H)$ are approximately equal to the complete spherical

\footnotetext{
Manuscript received by the Editor 29 November 2016; revised manuscript received 16 March 2017; published online 16 May 2017.

${ }^{1}$ Curtin University of Technology, Department of Spatial Sciences \& The Institute for Geoscience Research, Perth, Western Australia, Australia. E-mail: jack. mccubbine@curtin.edu.au;w.featherstone@curtin.edu.au; j.kirby@curtin.edu.au.

(C) 2017 Society of Exploration Geophysicists. All rights reserved.
} 
Bouguer anomaly (with the Bouguer slab given by $4 \pi G \rho H$ ), at least in regions without extreme elevation changes.

We use the $1^{\prime \prime} \times 1$ " DEM-H model provided by Geoscience Australia (Gallant et al., 2011) (Figure 1). It is derived from SRTM, has vegetation removed to convert the digital surface model to a DEM, is adaptively smoothed depending on the roughness of the topography and noise in the SRTM, and hydrological connectivity is enforced using the ANUDEM software (Hutchinson, 1989). The 1" DEM-H file size is approximately 100 times larger than the 9" GEODATA DEM used previously, posing some computational challenges discussed below.

The gravimetric terrain correction $\Delta g_{\mathrm{TC}}$ evaluated at a point $(x, y)$ for roving terrain at points $\left(x^{\prime}, y^{\prime}\right)$ with an assumed-constant topographic density $\rho$ (topographic bulk density errors are not considered here) is calculated by evaluating the convolution integral

$$
\begin{aligned}
& \Delta g_{\mathrm{TC}}(x, y)=-G \rho \iint_{\Omega} \int_{H(x, y)}^{H^{\prime}\left(x^{\prime}, y^{\prime}\right)} \frac{H-z^{\prime}}{\left[l^{2}+\left(H-z^{\prime}\right)^{2}\right]^{1 / 2}} d x^{\prime} d y^{\prime} d z^{\prime} \\
& =-G \rho \iint_{\Omega} \frac{1}{\left[l^{2}+\left(H-H^{\prime}\right)^{2}\right]^{1 / 2}}-\frac{1}{l} d x^{\prime} d y^{\prime} \\
& =G \rho \iint_{\Omega}\left\{\frac{1}{l}\left[1-\left[1+\left(\frac{H-H^{\prime}}{l}\right)^{2}\right]^{-1 / 2}\right]\right\} d x^{\prime} d y^{\prime}
\end{aligned}
$$

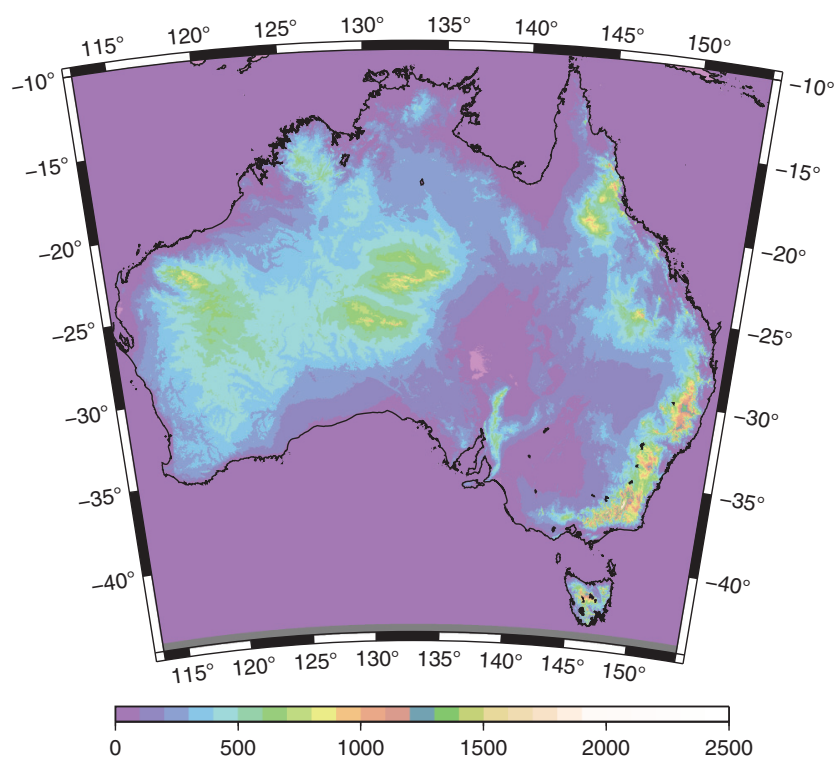

Figure 1. The DEM-H model (meters) of Australia (Lambert conic conformal projection). (Max: $2223.25 \mathrm{~m}$, min: $-68.05 \mathrm{~m}$, mean: $138.03 \mathrm{~m}$, and STD: $\pm 194.38 \mathrm{~m}$ ).

Table 1. Descriptive statistics of the DEM-H gradients (arc-degrees) and number of cases $>45$ arc-degrees. There are 18,330,863,392 elements in DEM-H.

\begin{tabular}{lcccccc}
\hline Gradient & Max & Min & Mean & STD & $\#>45^{\circ}$ & $\%$ \\
\hline North/south & $81^{\circ}$ & $-81^{\circ}$ & $0^{\circ}$ & $2^{\circ}$ & 61,077 & 0.000333 \\
West/east & $83^{\circ}$ & $-82^{\circ}$ & $0^{\circ}$ & $2^{\circ}$ & 97,032 & 0.000529 \\
North-west/south-east & $84^{\circ}$ & $-84^{\circ}$ & $0^{\circ}$ & $2^{\circ}$ & 75,982 & 0.000415 \\
North-east/South-west gradient & $84^{\circ}$ & $-83^{\circ}$ & $0^{\circ}$ & $2^{\circ}$ & 81,086 & 0.000442 \\
\hline
\end{tabular}

where $G=(6.67408 \pm 0.00031) \times 10^{-11} \mathrm{~m}^{3} \mathrm{~kg}^{-1} \mathrm{~s}^{-2}$ is the universal gravitational constant (Mohr et al., 2016), $H$ and $H^{\prime}$ are heights relative to the geoid at the computation and roving points, respectively, and the planar distance between the computation $(x, y)$ and roving $\left(x^{\prime}, y^{\prime}\right)$ points is

$$
l=\sqrt{\left(x^{\prime}-x\right)^{2}+\left(y^{\prime}-y\right)^{2}} .
$$

The binomial expansion of the inner term in equation 1 given as

$$
\begin{aligned}
& {\left[1+\left(\frac{H-H^{\prime}}{l}\right)^{2}\right]^{-1 / 2}=1-\frac{1}{2}\left(\frac{H-H^{\prime}}{l}\right)^{2}} \\
& +\frac{3}{8}\left(\frac{H-H^{\prime}}{l}\right)^{4}-\frac{5}{16}\left(\frac{H-H^{\prime}}{l}\right)^{6} \ldots
\end{aligned}
$$

leads to a series expansion of the form

$$
\begin{aligned}
& \Delta g_{\mathrm{TC}}(x, y)=G \rho \iint_{\Omega} \frac{\left(H^{\prime}-H\right)^{2}}{2 l^{3}}-\frac{3\left(H^{\prime}-H\right)^{4}}{8 l^{5}} \\
& +\frac{5\left(H^{\prime}-H\right)^{6}}{16 l^{7}} \ldots d x^{\prime} d y^{\prime} .
\end{aligned}
$$

Martinec et al. (1996) and Tsoulis (2001) contend that this binomial expansion converges, provided that the topographic gradient does not exceed 45 arc-degrees. As such, we calculated (Table 1) and plotted (Figure 2) the locations where the DEM-H gradients exceed $45^{\circ}$, showing them to be very rare $(\sim 0.0005 \%$ of all DEM elements $)$ and restricted to the mountainous (maximum height $2223.25 \mathrm{~m}$ ) Great Dividing Range in eastern Australia, the southern island of Tasmania, and the coastline where there are cliffs (cf. Figure 1).

In the relatively flat terrain over most of Australia, and to demonstrate the algorithms, we consider it sufficient to compute the first-order term only, so that the planar terrain correction is truncated to (e.g., Moritz, 1968; Li and Sideris, 1994)

$$
\Delta g_{\mathrm{TC}}(x, y)=\frac{G \rho}{2} \iint_{\Omega} \frac{\left(H^{\prime}-H\right)^{2}}{l^{3}} d x^{\prime} d y^{\prime} .
$$

At points at which the DEM-H gradients exceed 45 arc-degrees, we removed the terrain correction values and error estimates, and we replaced them with the values interpolated from nearby.

The area that the integral in equation 5 is evaluated over is denoted by $\Omega$, such that $\left(x^{\prime}, y^{\prime}\right) \in \Omega$ if $0<l \leq R$, where $R$ is the maximum radius of integration beyond which the contribution to the terrain correction of topographic variations from the Bouguer plate is negligible. Empirical tests performed by Kirby and Featherstone (1999) found $R=50 \mathrm{~km}$ for Australia. For a $1^{\prime \prime}$ grid, the terrain correction omits near-gravimeter terrain effects out to approximately 30 m (cf. Leaman, 1998).

The brute-force numerical integration of the 10,178,544 elements inside $\Omega$ for $R=50 \mathrm{~km}$ is impractical, especially when Fourier methods have already proven themselves for terrain correction computations (e.g., Forsberg, 1985; Sideris, 1985; Li and Sideris, 1994; Parker, 1995, 1996). Therefore, we first expand the integral in equation 5 as 


$$
\begin{aligned}
& \Delta g_{\mathrm{TC}}(x, y)=\frac{G \rho}{2}\left[H^{2} \iint_{\Omega} \frac{1}{l^{3}} d x^{\prime} d y^{\prime}\right. \\
& \left.-2 H \iint_{\Omega} \frac{H^{\prime}}{l^{3}} d x^{\prime} d y^{\prime}+\iint_{\Omega} \frac{H^{\prime 2}}{l^{3}} d x^{\prime} d y^{\prime}\right],
\end{aligned}
$$

and we apply the 2D convolution theorem, which takes the sum of convolutions into the Fourier domain to become a sum of products, such that for constant grid spacings $\Delta x^{\prime}$ and $\Delta y^{\prime}$, we obtain

$$
\begin{aligned}
& \Delta g_{\mathrm{TC}}(x, y) \\
& \quad=\frac{G \rho}{2}\left[H^{2}\left(1 * \frac{1}{l^{3}}\right)-2 H\left(H^{\prime} * \frac{1}{l^{3}}\right)+\left(\frac{1}{l^{3}} * H^{\prime 2}\right)\right] \Delta x^{\prime} \Delta y^{\prime} .
\end{aligned}
$$

We implement the convolutions using the fast-Fourier transform, where for practical implementation in computer code, $f * g=$ $\mathbf{F}^{-1}[\mathbf{F}[f] \mathbf{F}[g]]$.

\section{Computational considerations}

The entire 1 " $\times 1$ " DEM-H model occupies $80 \mathrm{~Gb}$ in ASCII format, which is too large to handle in a single computation without a supercomputer. To permit computations on a PC, we divided the DEM-H into 90 overlapping square tiles, each containing 18,001 $\times$ 18,001 elements. The overlap was set at $R=50 \mathrm{~km}$ to eliminate edge effects. We computed the planar terrain corrections for each tile using equation 7 , and then we merged these together along the midpoints of the overlap (Figure 3).

Even for this 90-tile DEM, the FORTRAN77 code used by Kirby and Featherstone $(1999,2002)$, which used Netlib's IMSL fft3d routine, was limited by the maximum number of integers that the GNU compiler could handle on the LINUX server as described below. Therefore, we wrote a MATLAB script so as to make use of the fastest Fourier transform in the West (FFTW) algorithm, which adapts itself to make best use of the PC hardware (Frigo and Johnson, 2005).

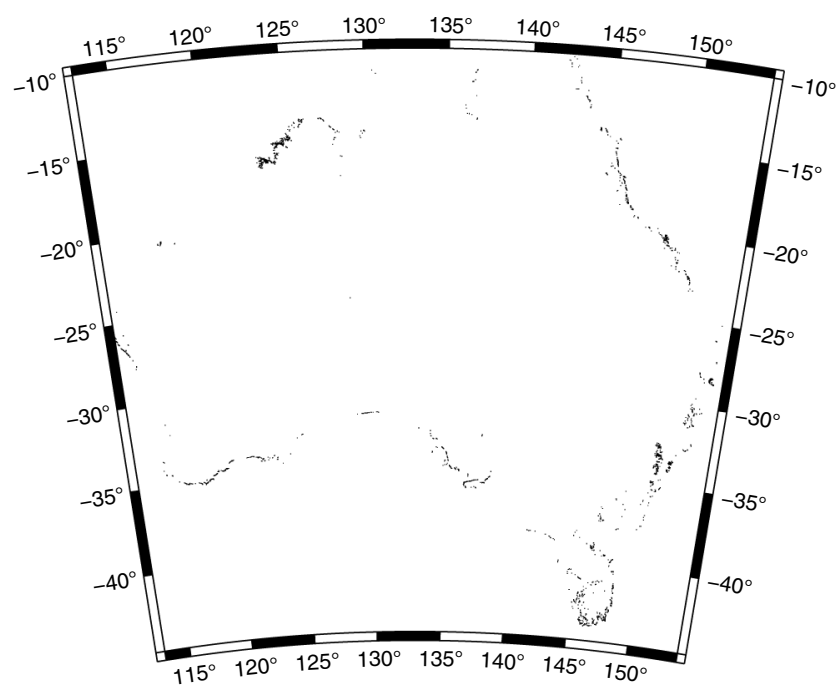

Figure 2. Composite map of locations where the DEM-H gradients exceed 45 arc-degrees.
We used an $18,001 \times 18,001$ subset of DEM-H to determine the efficient gains achieved by using the FFTW over $\mathrm{ft} 3 \mathrm{~d}$. Using the FORTRAN77 $\mathrm{fft} 3 \mathrm{~d}$ code on a LINUX server [40 Intel Xeon E5-2690 v2 @ $3.00 \mathrm{GHz}$ CPUs, with 347 Gb of RAM running Red Hat Enterprise Linux Server release 6.7] took approximately $9 \mathrm{~h}$, whereas using MATLAB FFTW on a PC [Intel Core i74770 CPU @ $3.40 \mathrm{GHz}$ (8 CPUs), approximately $3.4 \mathrm{GHz}$, with $16 \mathrm{~Gb}$ of RAM running Windows 7 Enterprise 64-bit] took approximately15 min, a 36-fold increase in computational (wall clock) speed. Additionally, equations 7, 12, 13, and 16 contain multiple instances of the same Fourier transforms, so these need only be computed once.

We used a kernel weighting for the difference between the mean value over each cell and the value at its center to improve the accuracy of the numerical integration. For equal grid spacings $\Delta x=\Delta y$, the kernel weighting in Featherstone and Olliver (1997) simplifies to

$$
w=\frac{2 l^{4}}{(l+\Delta x / 2)^{2}(l-\Delta x / 2)^{2}} .
$$

\section{ERROR PROPAGATION}

\section{Uncorrelated height errors}

We first consider the case of uncorrelated DEM height errors $\varepsilon_{H^{\prime}}$ at every roving cell in DEM-H. The $(x, y)$ and $\left(x^{\prime}, y^{\prime}\right)$ terms are taken to be error-free because these are grid registrations and not measurements. The contribution of a single DEM element with volume $\left(H^{\prime}-H\right) \Delta x^{\prime} \Delta y^{\prime}$ (Figure 4) to the planar terrain correction is

$$
\Delta g_{\mathrm{TC} 1}(x, y)=\frac{G \rho}{2} \frac{\left(H^{\prime}-H\right)^{2}}{l^{3}} \Delta x^{\prime} \Delta y^{\prime} .
$$

The height difference error is given by $\varepsilon_{\left(H^{\prime}-H\right)}=\sqrt{\varepsilon_{H}^{2}+\varepsilon_{H^{\prime}}^{2}}$ (Figure 4). Using a first-order Taylor series expansion, the height

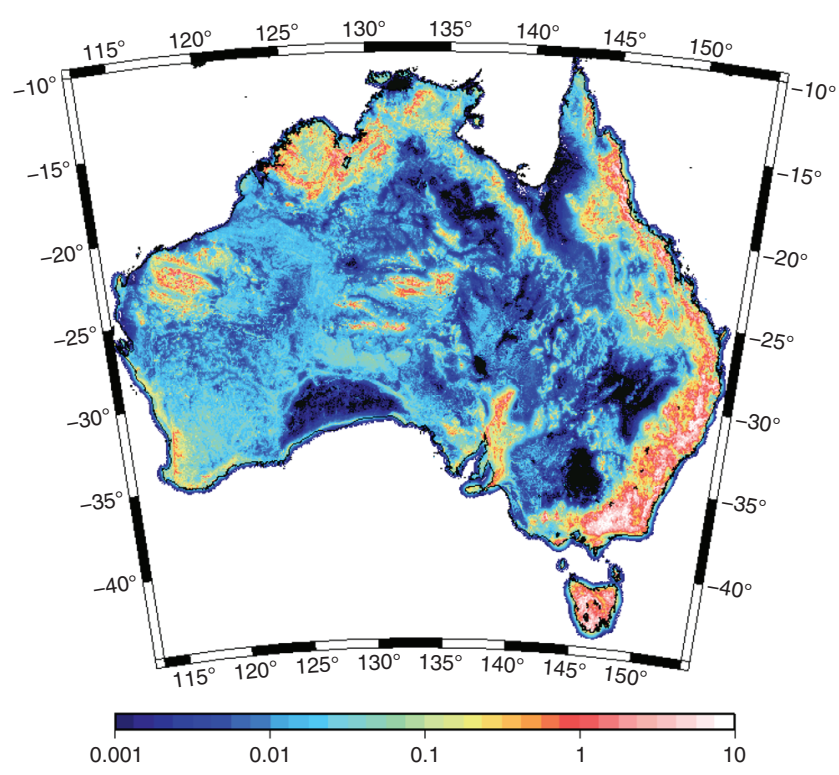

Figure 3. The planar gravimetric terrain corrections (mGal) over Australia. (Max: $51.550 \mathrm{mGal}$, min: $0 \mathrm{mGal}$, mean: $0.096 \mathrm{mGal}$, and STD: $\pm 0.580 \mathrm{mGal})$. 
difference error contributes to an error in the terrain correction of the form

$$
\varepsilon_{\mathrm{TC} 1}(x, y) \approx \frac{\partial \Delta g_{\mathrm{TC} 1}}{\partial\left(H^{\prime}-H\right)} \varepsilon_{\left(H^{\prime}-H\right)} .
$$

If we assume independence of the DEM heights in each cell, the total error variance in the terrain correction $\varepsilon_{\mathrm{TC}}(x, y)$ is given as

$$
\varepsilon_{\mathrm{TC}}(x, y) \approx \sqrt{\sum_{\Omega}\left[\left(\varepsilon_{H^{\prime}}^{2}+\varepsilon_{H}^{2}\right)\left(\frac{\partial \Delta g_{\mathrm{TC} 1}}{\partial\left(H^{\prime}-H\right)}\right)^{2}\right]} .
$$

As we did for the planar terrain correction values, 2D Fourier transforms are used to evaluate the error convolution efficiently, so that for each grid point

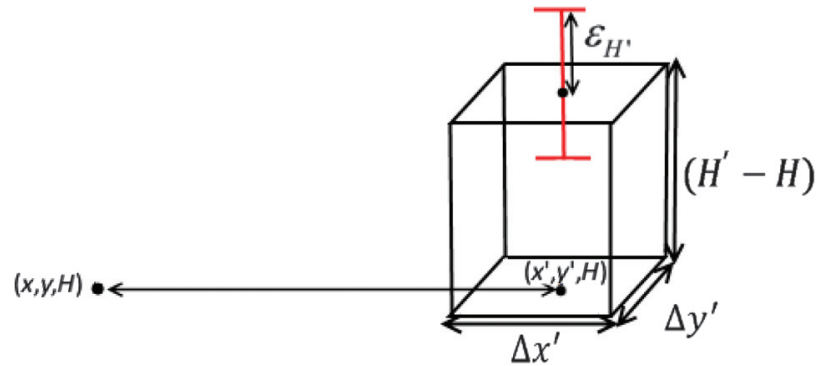

Figure 4. Geometry of the terrain correction for a single DEM element $\left(H^{\prime}-H\right) \Delta x^{\prime} \Delta y^{\prime}$ and the height difference error $\varepsilon_{\left(H^{\prime}-H\right)}=$ $\sqrt{\varepsilon_{H}^{2}+\varepsilon_{H^{\prime}}^{2}}$.

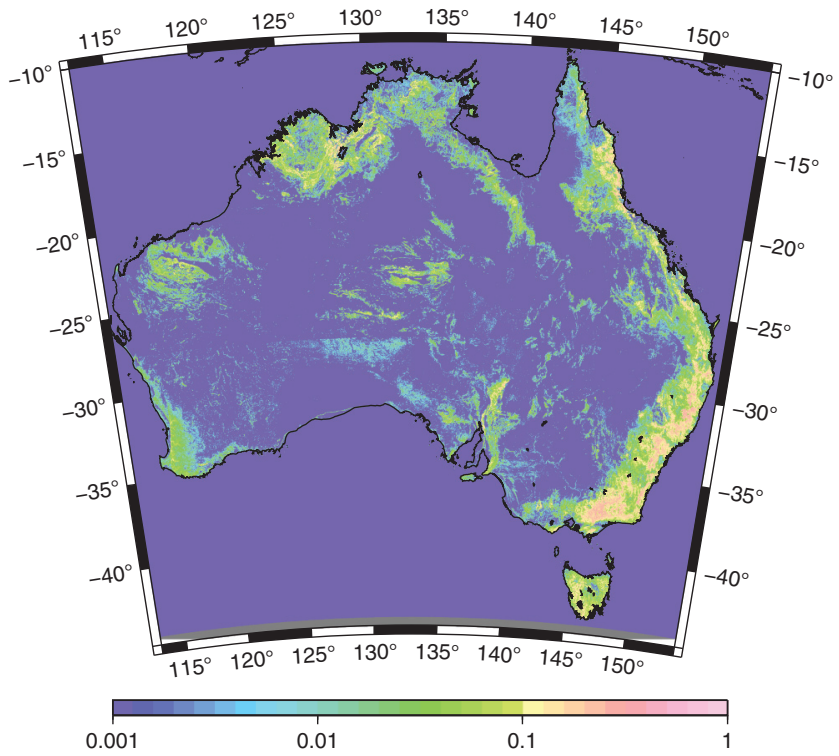

Figure 5. Standard deviation of error in the planar terrain correction (mGal) over Australia from equation 13 for constant and uncorrelated DEM errors of $\pm 7.66 \mathrm{~m}$. Max: $2.107 \mathrm{mGal}$, min: $0.000 \mathrm{mGal}$, mean: 0.000(3) mGal, and STD: $\pm 0.022 \mathrm{mGal}$.

$$
\begin{aligned}
& \varepsilon_{\mathrm{TC}}(x, y) \\
& =G \rho\left[\begin{array}{l}
H^{2}\left(\varepsilon_{H^{\prime}}^{2} * \frac{1}{l^{6}}\right)-2 H\left(H^{\prime} \varepsilon_{H^{\prime}}^{2} * \frac{1}{l^{6}}\right)+\left(H^{\prime 2} \varepsilon_{H^{\prime}}^{2} * \frac{1}{l^{6}}\right) \\
+\varepsilon_{H}^{2} H^{2}\left(1 * \frac{1}{l^{6}}\right)-2 \varepsilon_{H}^{2} H\left(\frac{1}{l^{6}} * H^{\prime}\right)+\varepsilon_{H}^{2}\left(H^{\prime 2} * \frac{1}{l^{6}}\right)
\end{array}\right]^{1 / 2} \Delta x^{\prime} \Delta y^{\prime} .
\end{aligned}
$$

Equation 12 requires a distinct error value per every DEM element, which is rarely available. In the interim, we adopt a "universal" error value that is the same for every DEM element. The height difference error values are then given by $\varepsilon_{\left(H^{\prime}-H\right)}=\sqrt{\varepsilon_{H}^{2}+\varepsilon_{H^{\prime}}^{2}}=\sqrt{2} \times \varepsilon_{H^{\prime}}$ and writing equation 11 as a convolution in the Fourier domain gives

$$
\begin{aligned}
& \varepsilon_{\mathrm{TC}}(x, y)=\sqrt{2} G \rho\left[H^{2}\left(\varepsilon_{H^{\prime}}^{2} * \frac{1}{l^{6}}\right)-2 H\left(H^{\prime} \varepsilon_{H^{\prime}}^{2} * \frac{1}{l^{6}}\right)\right. \\
& \left.+\left(H^{\prime 2} \varepsilon_{H^{\prime}}^{2} * \frac{1}{l^{6}}\right)\right]^{1 / 2} \Delta x^{\prime} \Delta y^{\prime} .
\end{aligned}
$$

One approach to estimate errors in DEMs is to compare the gridded data with an independent data set such as leveling (e.g., Hirt et al., 2010) to give a universal estimate of the DEM height accuracy for all grid cells. The elevation error in the DEM-H model is difficult to characterize, but Gallant et al. (2011) indicate that $90 \%$ of the DEM elements are within $9.8 \mathrm{~m}$ of leveled heights. Gallant et al. (2011) also report that "significant changes to elevation have occurred due to the smoothing and drainage enforcement processes, ... errors as large as $200 \mathrm{~m}$ occur in some areas." This $90 \%$ is approximately 1.28 standard deviations, given a Gaussian distribution, so we took $\varepsilon_{H^{\prime}}=9.8 / 1.28 \approx \pm 7.66 \mathrm{~m}$ as the universal standard deviation of the DEM-H height errors to generate Figure 5 using equation 13. Should a per-element error model become available, then equation 12 should be used in preference.

\section{Correlated height errors}

It is unrealistic to assume that the height errors for each grid cell in a DEM are independent and uncorrelated. Generally, DEM errors are highly correlated among neighboring cells in the near field. For instance, Gallant et al. (2011) and Rodríguez et al. (2006) indicate that the high-frequency SRTM DEM errors may be spatially correlated over horizontal distances $l$ from 0 to $100-400 \mathrm{~m}$.

This is of importance for terrain correction error estimates because the near-zone height differences $\left(H^{\prime}-H\right)$ contribute more to the terrain correction than those of the far zone (equation 5). However, the $\left(H^{\prime}-H\right)$ term in the numerator of equation 5 means that any correlated errors between the computation and roving points will cancel, which is not taken into account in the uncorrelated error case (equations 12 and 13).

To account for the cancelation of correlated DEM errors, we propose an exponential semivariogram (Figure 6), though any other functional model could be chosen. We choose this form so that the decorrelation can be set for any distance from the computation point. We write the height difference error variance $\varepsilon_{\left(H^{\prime}-H\right)}^{2}$ as a function of $l$ as a semivariogram with a range $a$ (beyond which the DEM height difference error variances become uncorrelated) and the uncorrelated height error variance value of $\sigma_{H^{\prime}}^{2}=(7.66 \mathrm{~m})^{2}=$ $58.68 \mathrm{~m}^{2}$ such that 


$$
\varepsilon_{\left(H^{\prime}-H\right)}^{2}=2 \sigma_{\left(H^{\prime}\right)}^{2}\left(1-e^{-3 l / a}\right) .
$$

Applying equation 13 to equation 11 gives

$$
\varepsilon_{\mathrm{TC}}(x, y)^{2}=\sum_{\Omega} 2 \sigma_{\left(H^{\prime}\right)}^{2}\left(1-e^{-3 l / a}\right)\left(\frac{\partial \Delta g_{\mathrm{TC} 1}}{\partial\left(H^{\prime}-H\right)}\right)^{2}
$$

Equation 15 can be written as a sum of convolutions similarly to equation 13 and computed in the 2D Fourier domain to obtain terrain correction error estimates efficiently, where the height error contribution of cells in the DEM closer to the computation point is reduced; i.e.,

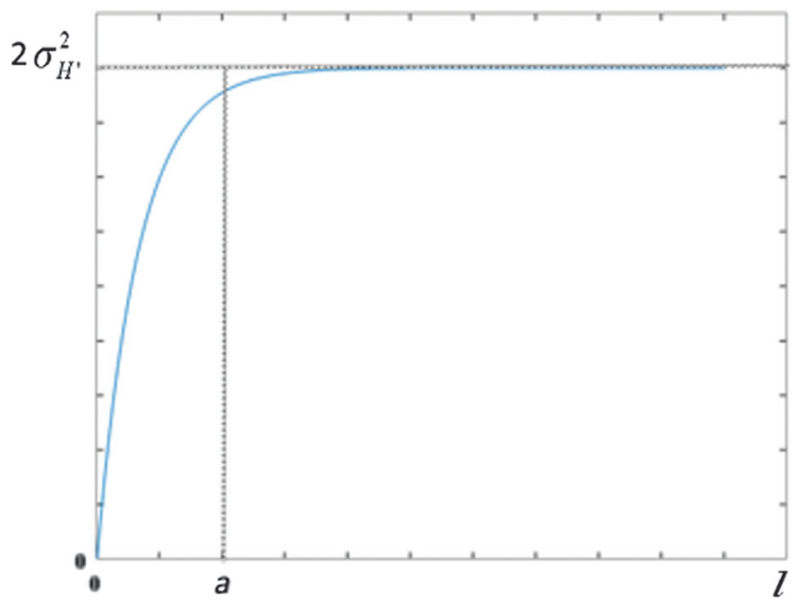

Figure 6. Exponential semivariogram. At $l=a, \varepsilon_{\left(H^{\prime}-H\right)}^{2}(l)$ is $95 \%$ the value of $2 \sigma_{H^{\prime}}^{2}$

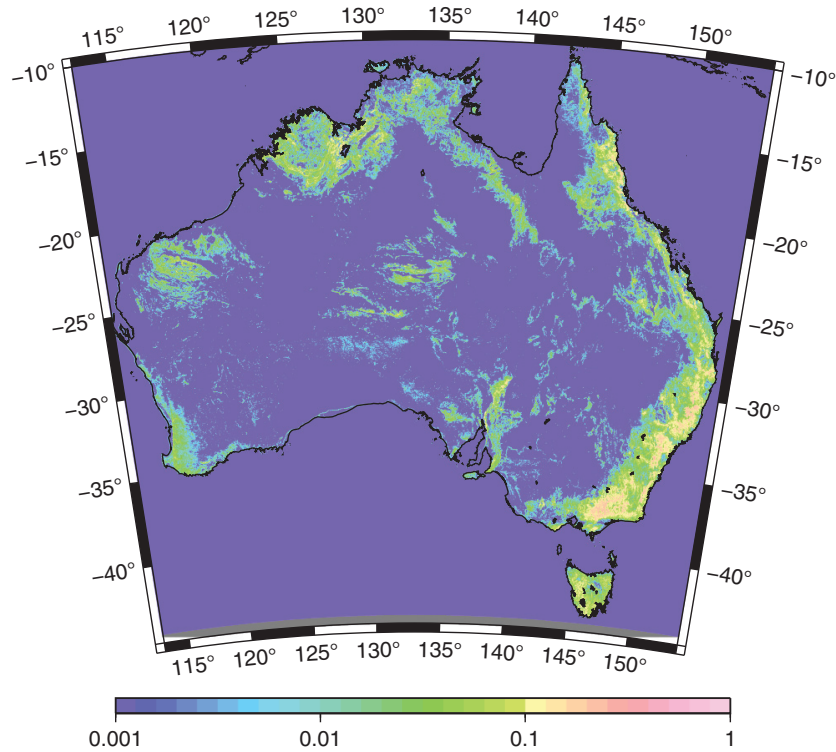

Figure 7. Standard deviation of error in the planar terrain correction (mGal) over Australia from equation 16 for correlated height errors with $a=100 \mathrm{~m}$ and $\sigma_{H^{\prime}}^{2}=58.68 \mathrm{~m}^{2}$. Max: $1.678 \mathrm{mGal}$, min: $0.000 \mathrm{mGal}$, mean: 0.000(2) $\mathrm{mGal}$, and STD: $\pm 0.018 \mathrm{mGal}$.

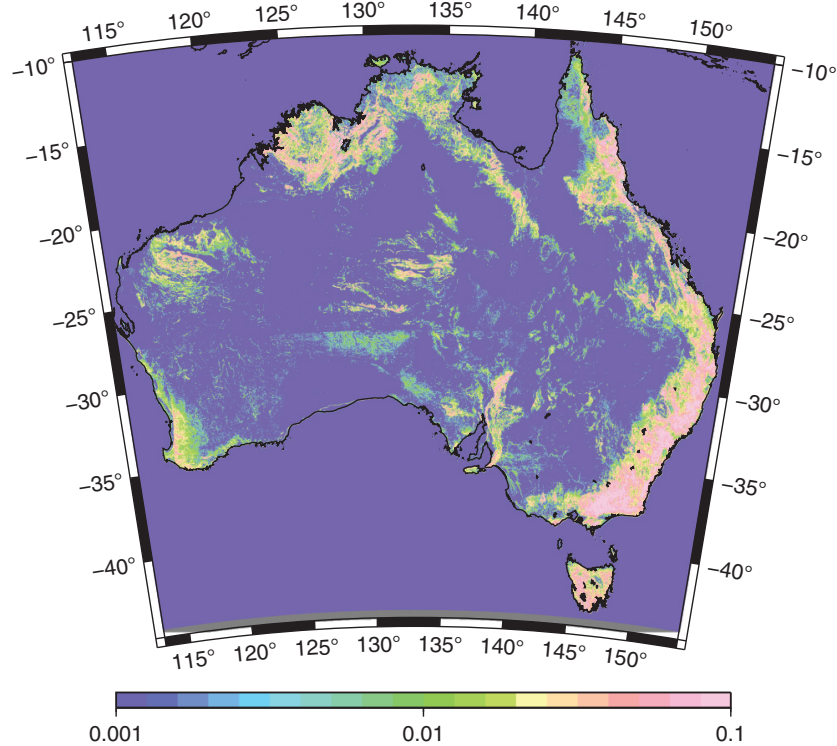

Figure 8. Differences between Figures 5 and 7. Max: $0.448 \mathrm{mGal}$, min: $0.000 \mathrm{mGal}$, mean: $0.000 \mathrm{mGal}$, and STD: $\pm 0.005 \mathrm{mGal}$.

$$
\begin{aligned}
& \varepsilon_{\mathrm{TC}}(x, y) \\
& =\sqrt{2} G \rho\left[\begin{array}{l}
H^{2}\left\{\left(\sigma_{H^{\prime}}^{2} * \frac{1}{l^{6}}\right)-\left(\sigma_{H^{\prime}}^{2} * \frac{e^{-3 l l a}}{l^{6}}\right)\right\}- \\
2 H\left\{\left(H^{\prime} \sigma_{H^{\prime}}^{2} * \frac{1}{l^{6}}\right)-\left(H^{\prime} \sigma_{H^{\prime}}^{2} * \frac{e^{-3 l / a}}{l^{6}}\right)\right\} \\
+\left(H^{\prime 2} \sigma_{H^{\prime}}^{2} * \frac{1}{l^{6}}\right)-\left(H^{\prime 2} \sigma_{H^{\prime}}^{2} * \frac{e^{-3 l / a}}{l^{6}}\right)
\end{array}\right]^{1 / 2} \Delta x
\end{aligned}
$$

The error in the terrain correction is reduced when the exponential semivariogram is included to account for the cancelation of correlated DEM errors close to each computation point (Figures 7 and 8).

\section{CONCLUSIONS}

A gap exists in the literature on the propagation of DEM height errors in the computation of gravimetric terrain corrections. We have therefore derived error propagation formulas for uncorrelated and correlated cases, using the planar terrain correction model as an example. Although the planar terrain correction (equation 5) is suitable for Australia, with only $0.0005 \%$ of the DEM-H gradients greater than 45 arc-degrees (Figure 2 and Table 1), it might not be suitable for densely sampled DEMs in more rugged terrains. All computations in the 2D Fourier domain via the FFTW algorithm show significant efficiency gains on a moderately powered PC with MATLAB installed.

We have presented the highest resolution $\left(1^{\prime \prime}\right.$ or $\left.\sim 30 \mathrm{~m}\right)$ grid of planar terrain corrections over the whole of Australia to date. It includes per-element error estimates from an exponential semivariogram to account for the cancelation of correlated errors that occurs due to the $\left(H^{\prime}-H\right)$ term in the numerator of equation 5. Figures 5, 7 , and 8 demonstrate that almost half of the power of the error results from erroneous topographic variations out to $100 \mathrm{~m}$ from each computation point. 
Even though our mathematical derivations are given for the planar terrain correction, they can be applied to other terrain-correction algorithms and geometries (e.g., spherical and ellipsoidal terrain corrections, spatial-domain calculations, or terrain elements with a different geometry). In our numerical examples for Australia, we have had to make some compromises because of the lack of (1) perelement height error estimates in the DEM-H and (2) exact knowledge of the correlation length of these DEM errors. Therefore, we recommend these additional data be sourced to provide more reliable error estimates in the gravimetric terrain correction.

\section{ACKNOWLEDGMENTS}

This work has been supported financially by the Cooperative Research Centre for Spatial Information, whose activities are funded by the Business Cooperative Research Centres Programme, and by Geoscience Australia. We thank Geoscience Australia for providing the DEM-H model, the associate editor X. Li, L. Wu, and two other anonymous reviewers for their constructive critiques.

\section{REFERENCES}

Dransfield, M., and Y. Zeng, 2009, Airborne gravity gradiometry: Terrain corrections and elevation error: Geophysics, 74, no. 5, I37-I42, doi: 10.1190/1.3170688.

Farr, T. G., P. A. Rosen, E. Caro, R. Crippen, R. Duren, S. Hensley, M. Kobrick, M. Paller, E. Rodriguez, L. Roth, D. Seal, S. Shaffer, J. Shimada, J. Umland, M. Werner, M. Oskin, D. Burbank, and D. Alsdorf, 2007, The shuttle radar topography mission: Reviews of Geophysics, 45, RG2004, doi: 10.1029/2005RG000183.

Featherstone, W. E., and J. G. Olliver, 1997, A method to validate gravimetric geoid computation software based on Stokes's integral: Journal of Geodesy, 71, 571-576, doi: 10.1007/s001900050125.

Forsberg, R., 1985, Gravity field terrain effect computations by FFT: Bulletin Géodésique, 59, 342-360, doi: 10.1007/BF02521068.

Frigo, M., and S. G. Johnson, 2005, The design and implementation of FFTW3: Proceedings of the IEEE, 93, 216-231, doi: 10.1109/JPROC .2004.840301.

Gallant, J. C., T. I. Dowling, A. M. Read, N. Wilson, P. Tickle, and C. Inskeep, 2011, 1 second SRTM derived digital elevation models user guide: Geoscience Australia www.ga.gov.au/topographic-mapping/digital-elevationdata.html, http://www.ga.gov.au/metadata-gateway/metadata/record/gcat 72759, accessed 16 January 2016

Hirt, C., M. S. Filmer, and W. E. Featherstone, 2010, Comparison and validation of recent freely-available ASTER-GDEM ver1, SRTM ver4.1 and GEODATA DEM-9S ver3 digital elevation models over Australia: Australian Journal of Earth Sciences, 57, 337-347, doi: 10.1080/ 08120091003677553.
Hutchinson, M. F., 1989, A new procedure for gridding elevation and stream line data with automatic removal of spurious pits: Journal of Hydrology, 106, 211-232, doi: 10.1016/0022-1694(89)90073-5.

Kirby, J. F., and W. E. Featherstone, 1999, Terrain correcting Australian gravity observations using the national digital elevation model and the fast Fourier transform: Australian Journal of Earth Sciences, 46, 555-562, doi: 10.1046/j.1440-0952.1999.00731.x.

Kirby, J. F., and W. E. Featherstone, 2002, High-resolution grids of gravimetric terrain correction and complete Bouguer corrections over Australia: Exploration Geophysics, 33, 161-165, doi: 10.1071/EG02161.

Kuhn, M., W. E. Featherstone, and J. F. Kirby, 2009, Complete spherical Bouguer gravity anomalies over Australia: Australian Journal of Earth Sciences, 56, 213-223, doi: 10.1080/08120090802547041.

Leaman, D. E., 1998, The gravity terrain correction practical considerations: Exploration Geophysics, 29, 467-471, doi: 10.1071/EG998467.

Li, Y. C., and M. G. Sideris, 1994, Improved gravimetric terrain corrections: Geophysical Journal International, 119, 740-752, doi: 10.1111/j.1365246X.1994.tb04013.x.

Malgorzata, S., and J. Krynski, 2009, Evaluation of the effect of uncertainty of height data on the accuracy of terrain corrections: Geodesy and Cartography, 58, 71-88.

Martinec, Z., P. Vanícěk, A. Mainville, and M. Véronneau, 1996, Evaluation of topographical effects in precise geoid computation from densely sampled heights: Journal of Geodesy, 70, 746-754, doi: 10.1007/BF00867153.

Mikuška, J., R. Pašteka, and I. Marušiak, 2006, Estimation of distant relief effect in gravimetry: Geophysics, 71, no. 6, J59-J69, doi: 10.1190/1.2338333.

Mohr, P. J., D. B. Newell, and B. N. Taylor, 2016, CODATA recommended values of the fundamental physical constants: 2014: Reviews of Modern Physics, 88, 035009, doi: 10.1103/RevModPhys.88.035009.

Moritz, H., 1968, On the use of the terrain correction in solving Molodensky's problem: Report 108, Department of Geodetic Science, Ohio State University.

Nowell, D. A. G., 1999, Gravity terrain corrections: An overview: Journal of Applied Geophysics, 42, 117-134, doi: 10.1016/S0926-9851(99) 00028-2.

Parker, R. L., 1995, Improved Fourier terrain correction — Part 1: Geophysics, 60, 1007-1017, doi: 10.1190/1.1443829.

Parker, R. L., 1996, Improved Fourier terrain correction — Part 2: Geophysics, 61, 365-372, doi: 10.1190/1.1443965.

Rodríguez, E., C. S. Morris, and J. E. Belz, 2006, A global assessment of the SRTM performance: Photogrammetric Engineering and Remote Sensing, 72, 249-260, doi: 10.14358/PERS.72.3.249.

Sideris, M. G., 1985, A fast Fourier transform method for computing terrain corrections: Manuscripta Geodaetica, 10, 66-73.

Steinhauser, P., B. Meurers, and D. Ruess, 1990, Gravity investigations in mountainous areas: Exploration Geophysics, 21, 161-168, doi: 10.1071/ EG990161.

Tsoulis, D., 2001, Terrain correction computations for a densely sampled DTM in the Bavarian Alps: Journal of Geodesy, 75, 291-307, doi: 10 .1007/s001900100176.

Wu, L., 2016, Efficient modelling of gravity effects due to topographic masses using the Gauss-FFT method: Geophysical Journal International 205, 160-178, doi: 10.1093/gii/ggw010.

Zahorek, P., R. Pašteka, and J. Papco, 2010, The estimation of errors in calculated terrain corrections in the Tatra Mountains: Contributions to Geophysics and Geodesy, 40, 323-350, doi: 10.2478/v10126-010-0014-4. 